\title{
BIOMETANIZACIÓN DE RESIDUO SÓLIDO URBANO: ESCALA DE LABORATORIO Y PLANTA PILOTO
}

\section{BIOMETHANIZATION OF SOLID URBAN WASTE URBAN: LABORATORY SCALEAND PILOT SCALE}

\section{Forster-Carneiro, T. ${ }^{1}$, Pérez, M. ${ }^{2}$; Romero, L.I. ${ }^{3}$}

Departamento de Ingeniería Química, Tecnología de Alimentos y Tecnologías del Medio Ambiente. Facultad de Ciencias del Mar y Ambientales. Universidad de Cádiz. Apdo. 40. 11510 Puerto Real (Cádiz), SPAIN. Tfno: 956016158. Fax: 956016411. e-mail: tania.forster@uca.es

\section{RESUMO}

O objetivo geral deste trabalho foi estabelecer um protocolo apropriado para estudos de partida e estabilização de processos de biometanização de resíduos sólidos urbanos (RSU), sobre condições secas $\left(20-30\right.$ sólidos totais) e termofílicas $\left(55^{\circ} \mathrm{C}\right.$ de temperatura), e transferível à escala industrial. O primeiro estudo foi conduzido para desenvolver e aplicar as diferentes técnicas analíticas apropriadas à caracterização do RSU. O segundo estudo tinha o propósito de estudar os procedimentos específicos de partida e estabilização dos processos de digestão anaeróbia seca e termofílica, usando a tecnologia de reator agitado (5 litros de capacidade) e planta piloto. Os resultados do reator em escala de laboratório mostram rápida partida (20-30 dias) e apropriada atividade degradativa. O sistema opera de acordo com o protocolo utilizado. Finalmente no estudo de planta piloto os resultados indicam que, um reator em escala industrial utilizando a tecnologia de digestão anaeróbia seca e termofílica e $30 \%$ de inóculo com lodo mesofilico procedente de uma Estação de Tratamento de Águas Residuárias (EDAR) no tratamento de RSU, podem conseguir uma rápida partida $\mathrm{e}$ estabilização do sistema e alta biodegradação da matéria orgânica.

Palavras-chave: Biometanização. Termofílica. Lixo urbano. Metano. Biomassa.

\section{ABSTRACT}

The general objective of the present work has been to establish an appropriate protocol to carry out the start up and stabilization of the process of biomethanization of municipal solid waste (MSW), under dry (20-30\% total solid) and thermophilic $\left(55^{\circ} \mathrm{C}\right.$ temperature) conditions, and transferable to industrial scale. The first study 
was conducted to develop and apply the different technical analytic for the appropriate characterization of the MSW. The second objective was defined to propose a specific procedure to carry out the start up and stabilization of the process of dry thermophilic anaerobic digestion, using the batch technology (reactor with 5.0 $\mathrm{L}$ total capacity) and pilot plant. The results of reactor laboratory scale showed a fast start up (20-30 days) and appropriate degradate activity. The system was operating according to the used protocol. Finally, in the study of pilot plant the results obtained indicate that, a experiment using dry thermophilic anaerobic digestion and 30\% of inoculation with digested sludge from Waste Water Treatment Plant,(under mesophilic conditions), with MSW treatment, could be see a fast start up and stabilization phase, higher biodegradation of the organic matter and fast stabilization of the system.

Key-words: Biometanization. Thermophilic. Solid waste. Methane. Biomass.

\section{INTRODUCCIÓN}

La problemática medioambiental a escala mundial se centra, actualmente, en dos importantes aspectos: la generación exponencial de residuos y el incremento de la emisión de gases que potencian el efecto invernadero provocando el denominado cambio climático. La generación de residuos está aumentando rápidamente debido al rápido crecimiento demográfico, el aumento de la población en los centros urbanos, la utilización de bienes materiales de rápido envejecimiento y el uso, cada vez más generalizado, de envases sin retorno, creando serios problemas de disposición. En España, de acuerdo con el Plan Nacional de Residuos (2000-2006), se generan aproximadamente 24 millones de toneladas de residuos sólidos urbanos (RSU) al año; es decir $1,2 \mathrm{~kg}$ por habitante al día, de los que un $40-45 \%$ son de naturaleza orgánica (INE, 2002). Cuanto a la producción de lodos frescos se estima que está comprendida entre 200 y $250 \mathrm{~g} /$ persona/día (residuo deshidratado) o aproximadamente $50 \mathrm{~g}$ materia seca/persona/día (HERNÁNDEZ, 2001). Además, se han registrado según el Ministerio de Agricultura, Pesca y Alimentación un total de 689.488t de lodos de EDAR, expresados en materia seca, lo que corresponde con unas 3.500.000t de residuo deshidratado. Asimismo, se prevé un aumento de esta producción como consecuencia del aumento del número de EDARs en los próximos años debido al cumplimento de las exigencias de la Directiva 91/217/CEE, sobre tratamiento de aguas residuales urbanas.

En un panorama medioambiental y energético mundial, en los últimos años los avances técnicos han posibilitado la aparición de sistemas de aprovechamiento energético y de la biomasa cada vez más eficientes, fiables y limpios (HOUGHTON et al., 2001). Así, el gobierno español proyecta un aumento del potencial energético a partir de la biomasa. En la práctica supone duplicar la obtención de biogás en digestores a partir de la biomasa con el fin de producir energía y reducir la contaminación del medio ambiente por los residuos sólidos (De BAERE, 2000). El biogás generado en 
la digestión anaerobia de los RSU, de los lodos de depuración de aguas urbanas, y de los residuos ganaderos presentan un gran potencial energético, ya que su componente principal es el metano y, por tanto, puede emplearse para producir energía térmica, eléctrica o en sistemas de cogeneración. De acuerdo con los informes de la Agencia Internacional de Energía (IEA, 2003) existen 400 plantas industriales de digestión anaerobia en todo el mundo tratando RSU y residuos industriales. Las nuevas plantas de biometanización son conocidas como TMB - Tratamiento Mecánico Biológico, y poseen capacidad para el tratamiento de residuos de distintos orígenes, tales como, residuos verdes, lodos frescos y residuos sólidos urbanos. En lo que se refiere a residuos urbanos, ganaderos y forestales, son aproximadamente 130 plantas distribuidas en Europa (93\%) y Asia (7\%) (BIOGAS WORKS, 2005). En España la difusión de esta tecnología ha sido muy rápida, representando, del 30 al 50\% de las nuevas instalaciones de tratamiento de residuos son de digestión anaerobia, existen 19 plantas de biometanización de gran escala que permiten tratar cada una 1100000 toneladas de RSU por año, situando al país en aquél con mayor capacidad y potencial de tratamiento de residuos urbanos de toda Europa (De BAERE, 2005). Más concretamente, en la provincia de Cádiz se ha construido la primera Planta de Biometanización de RSU de España, dotada de unidades de digestión anaerobia seca y compostaje aeróbico, y actualmente la planta se encuentra en fase de prueba del arranque del sistema.

Se pueden establecer diversas clasificaciones para la digestión anaerobia de vertidos o residuos con alta carga orgánica en función del rango de operación. La elección de la tecnología más adecuada dependerá de los parámetros operacionales elegidos: régimen de alimentación; etapas del proceso; contenido en sólidos y temperatura de operación. Los procesos de digestión anaerobia para el tratamiento de los RSU se han clasificado en sistemas con bajo contenido en sólidos o también denominados "húmedos" (porcentajes de sólidos totales inferiores a 10) y sistemas de alto contenido en sólidos o digestión anaerobia "seca" (sólidos totales superiores a 20\%) (HARTMANN AND AHRING, 2005). En Europa, la capacidad de los procesos de digestión anaerobia seca alcanza el 54\% del total de los procesos para el tratamiento del residuo sólido, pues presenta diversas ventajas tales como, bajos requerimientos de agua para la dilución del residuo, menores requerimientos para la deshidratación del residuo final y mayor producción de biogás por unidad de volumen del reactor (De BAERE, 2005). Además un alto porcentaje de las plantas industriales de tratamiento de RSU son del tipo termofilicas $\left(55^{\circ} \mathrm{C}\right)$. Los estudios demuestran que la digestión anaerobia en el rango termofílico aumenta la velocidad de conversión (alta eficacia de eliminación de los sólidos orgánicos y de destrucción de organismos patógenos) permitiendo bajos tiempos hidráulicos de retención y altas velocidades de carga. Sin embargo también puede disminuir la estabilidad del proceso y pequeñas oscilaciones de temperatura, dentro del rango termofílico tiene un significativo efecto sobre la estabilidad del proceso (ROMERO et al., 1990). Por todo ello, múltiples autores, (BOUALLAGUI et al., 2004; EL-FADEL y MASSOUD, 2001) afirman que según la estrategia de arranque se observan diferentes comportamientos iniciales del proceso que 
se traducen en mayores o menores periodos de tiempo necesarios para la puesta en marcha.

Por todo ello, los trabajos previos ponen de manifiesto que el proceso presenta una relativa complejidad y que se carece de estudios que detallen los procedimientos más adecuados para el arranque de los reactores, según el tipo de RSU, y otros aspectos tales como el tipo de inóculo más adecuado, las diferentes configuraciones del proceso, porcentaje de sólidos, etc. (BOLZONELLA et al., 2003). Este aspecto conlleva la necesidad de estudiar las características específicas del arranque más adecuadas para cada tipo de residuo en particular. El objetivo de este estudio fue seleccionar las condiciones operacionales más adecuadas para realizar la etapa de arranque y estabilización de bio-reactores anaerobios termofílicos y secos, y obtener un procedimiento apropiado para el desarrollo de la fase de arranque de la digestión anaerobia seca de RSU, en un reactor de tanque agitado a escala de laboratorio. Asimismo, se pretende comprobar la aplicabilidad del estudio y la posibilidad de transferencia tecnológica al sector industrial, mediante ensayos realizados a escala de planta piloto.

\section{MATERIAL Y MÉTODOS}

La mayor parte de los reactores anaerobios implantados actualmente, a escala industrial, para el tratamiento de residuos sólidos urbanos responden a la tecnología de tanque agitado monoetapa. Por ello, en este trabajo se desarrolla el estudio experimental del efecto de las variables de operación más significativas sobre la puesta en marcha de la digestión anaerobia seca utilizando esta tecnología. Los estudios realizados consideran: (1) el porcentaje de sólidos del residuo; (2) el porcentaje de inoculación; (3) y naturaleza del inóculo. En consecuencia en este trabajo se pretende establecer un protocolo de arranque y estabilización del proceso de digestión anaerobia termofílica seca de RSU que pueda ser extrapolable a escala de planta piloto.

\subsection{Selección y Pre-tratamientos de la Biomasa}

Se han utilizado dos residuos orgánicos: residuos sólido urbano y lodos de depuradora.

1- Fracción orgánica de residuos sólidos urbanos (FORSU): procedente de la Planta de Reciclaje y Compostaje "Las Calandrias", más concretamente del tromel de $30 \mathrm{~mm}$.

2- Lodo fresco (LODO): como fuente de inóculo se ha utilizado lodo mesofílico procedente de la recirculación de los digestores anaerobios (LODO) de la Estación de Depuradora de Aguas Residuales (EDAR) - Guadalete de Jerez de la Frontera (Cádiz).

La caracterización de residuos sólidos heterogéneos (residuos de restaurante, residuos sólidos urbanos, etc.) presenta como primer problema la obtención de una 
muestra representativa del mismo. Por ello, se realizó un muestreo sobre lotes homogeneizados y seleccionados al azar para obtener la muestra bruta inicial. Para el manejo posterior del residuo (preparación de las alimentaciones de reactores, etc.) y su caracterización analítica completa se requiere un pretratamiento que mejore su manejabilidad y homogeneidad. En el presente estudio, el pretratamiento y acondicionamiento consistió una separación manual de las fracciones orgánicas e inorgánicas; algunos materiales orgánicos (principalmente, papel), con el objetivo de obtener una fracción orgánica de fácil biodegradación. A continuación se realizaron secado de las muestras, en una habitación termostatizada a $55^{\circ} \mathrm{C}$ de temperatura durante 24 horas hasta alcanzar un grado de humedad del 10-20\%. La FORSU seca fue triturada hasta un tamaño de partícula de 1-2- $\mathrm{cm}$ (reactores tanque agitado de 5L) para su utilización en un reactor a escala de laboratorio (FORSTER-CARNEIRO et al., 2004a). Ninguno tratamiento ha sido aplicado en las muestras de FORSU (reactor planta piloto) y los LODOS mesofílicos.

\subsection{Equipos Utilizados para la Biometanización}

Fueron utilizados dos reactores discontinuos, a escala laboratorio y planta piloto, que permiten estudiar la evolución de las fases de arranque y estabilización del proceso de biometanización.

A- Reactor tanque agitado de 5,0 L (escala de laboratorio): el equipo consta de un reactor agitado calefactado mediante la recirculación de agua a través de una camisa termostatizada. Se utiliza un baño modelo PRECISTERM 6000142/6000389 SELECTA S.A. con capacidad para 5 litros de agua. El sistema posee, además, un sistema de controlador de $\mathrm{pH}$ con depósitos de hidróxido de sodio (SOSA $5 \mathrm{~N}$ ) y ácido acético; un sistema de recogida de biogás (mediante bolsas TEDLAR); sistema de Agitación compuesto por un motor de agitación modelo IKA EUROSTART Power Control P4 que permite la homogenización del residuo a una velocidad de 14-530 rpm y un una llave de descarga de $20 \mathrm{~mm}$ de paso de luz en la parte inferior utilizada para la toma de muestra (Figura 1).

B- Reactor tanque agitado de $300,0 \mathrm{~L}$ (escala planta piloto): el reactor consiste en un vaso de acero inoxidable con una capacidad total de $300 \mathrm{~L}$ y una capacidad útil de $180 \mathrm{~L}$ (radio 0,28 m; altura 1,2m). El sistema posee un sistema de agitación compuesto por un motor de agitación SIEMENS y diversas aspas de acero inoxidable dispuestas en horizontal y ligeramente inclinadas, en los dos sentidos del eje; un sistema de control de temperatura dotado de dos termómetros (termómetro analógico tipo termopar y termómetro digital); sistema de control y seguridad con una válvula de seguridad; y una abertura con cierre roscado por donde se puede tomar muestra del interior del reactor, introducir correctores de $\mathrm{pH}$ (ácido/base), o introducir la alimentación. El sistema posee, además: una electro-bomba centrífuga modelo EBARA PUMPS EUROPE S.A. (presión 8 bares), empleada para elevar y distribuir el agua del baño sobre la camisa termostatizada del reactor; y una salida de biogás 
con un dispositivo de toma de muestra y un contador de gas de la marca Milli, modelo PMMA/PC. La lectura del biogás se realiza mediante un sensor magnético que funciona con la presión que ejerce el gas (Figura 2).

Previamente al sellado de los reactores, se adicionó inóculo hasta alcanzar un porcentaje del 30\%. A continuación, se añadió agua destilada en cantidades adecuadas para que los residuos orgánicos alcanzaran un porcentaje en sólidos totales del $20 \%$, de acuerdo con trabajos previos (FORSTER-CARNEIRO et al., 2004b).

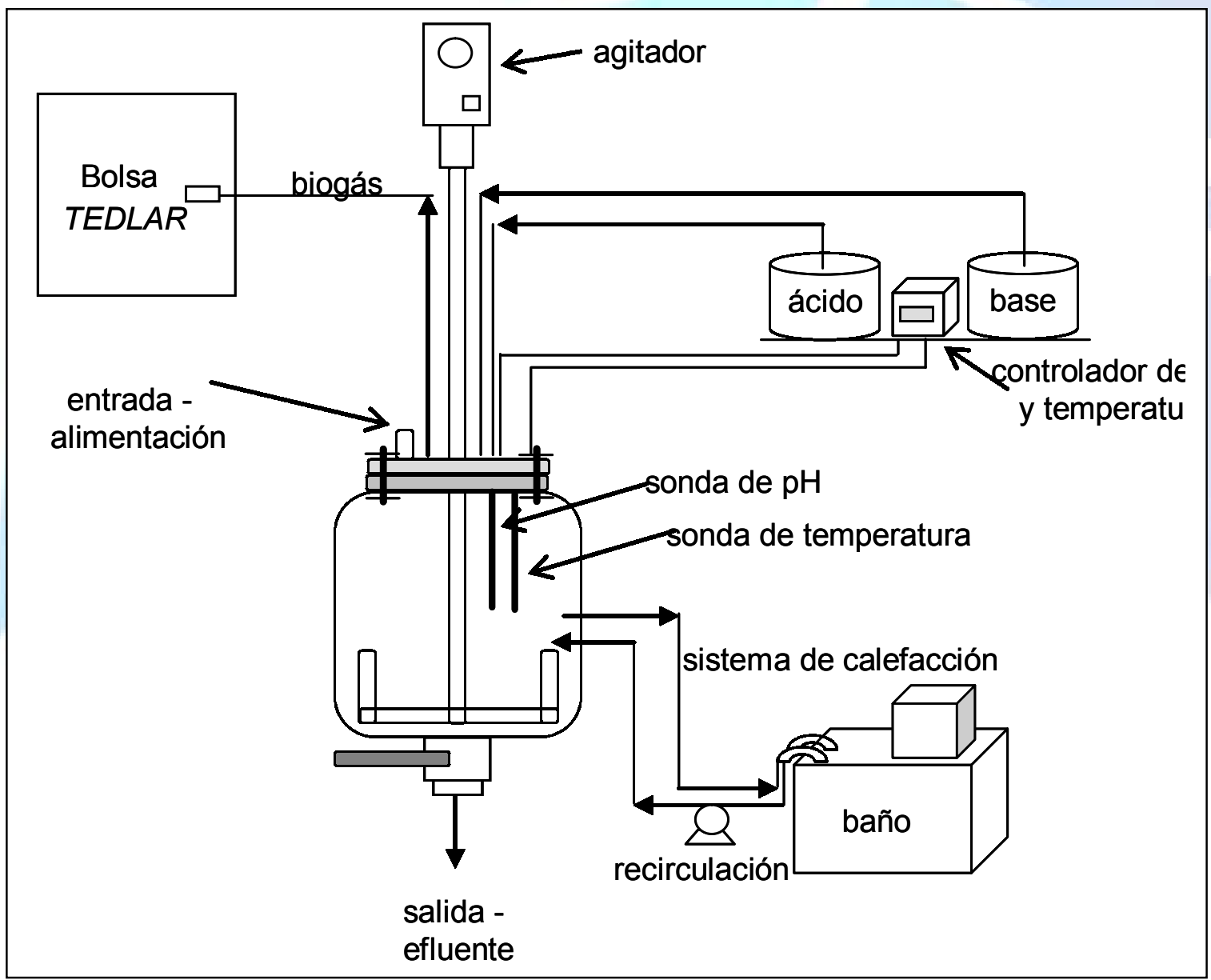

Figura 1. Reactor tipo tanque agitado discontinuo $(5,0 \mathrm{~L})$ a escala de laboratorio y esquema representativo. 


\begin{tabular}{|l|r|}
\hline Recebido em: 09/02/2007 & HOLOS Environment, v.7 n.1, 2007 - P. 93 \\
\hline Liberado para Publicação em: 15/12/2007 & ISSN:1519-8421 (CD-ROM) / ISSN:1519-8634 (ON-LINE) \\
\hline
\end{tabular}
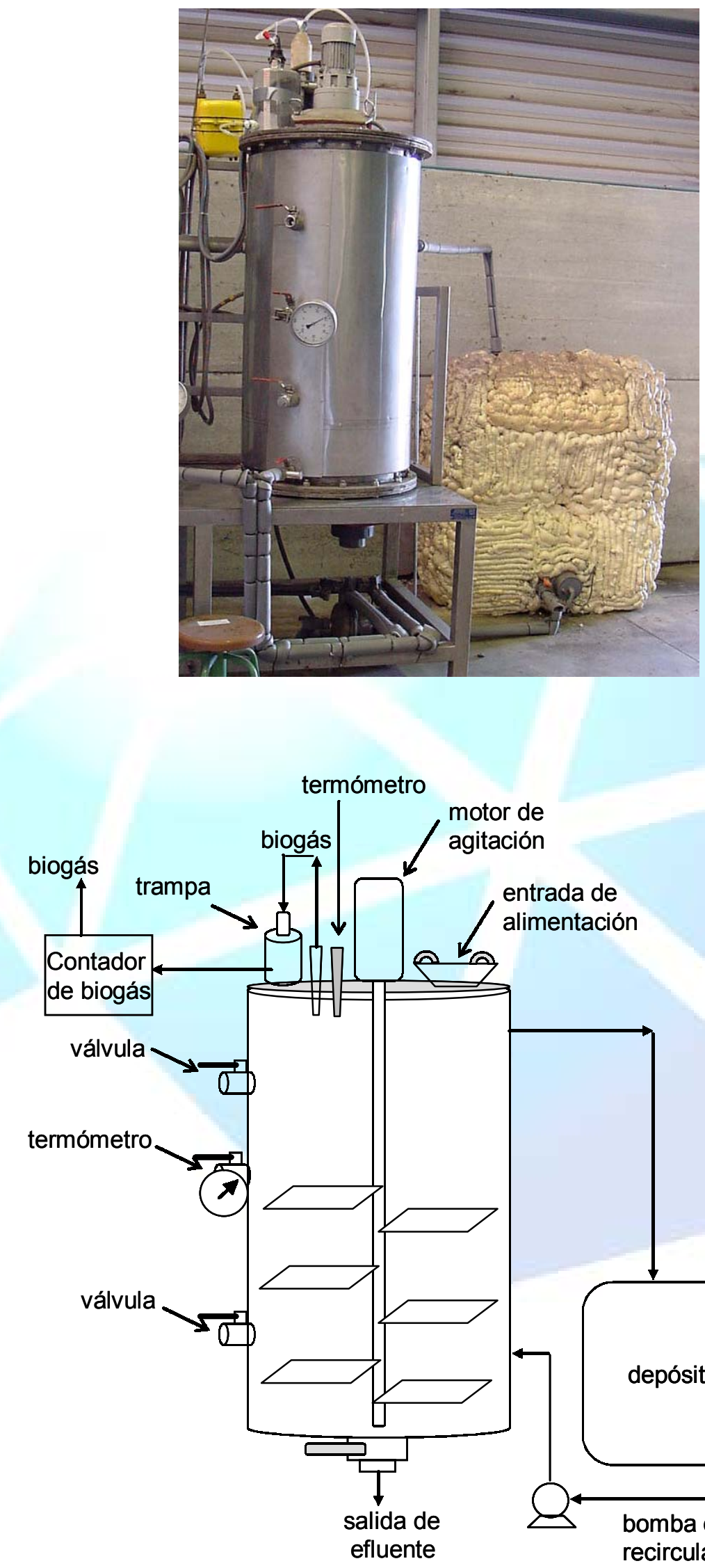

\section{motor de}

agitación 


\subsection{Técnicas Analíticas Utilizadas}

Para el seguimiento y control del proceso de biometanización del ROF se analizaron diariamente los parámetros más significativos del residuo y biogás. Los parámetros analizados en las muestras del residuo fueron: sólidos, $\mathrm{pH}$, alcalinidad, nitrógeno amoniacal ( $\mathrm{N}$-amon), demanda química de oxígeno (DQO), volumen y composición del biogás. Los sólidos están divididos en sólidos totales, en suspensión y disueltos, así los sólidos totales (ST) incluyen los sólidos totales en suspensión (STS) y los sólidos totales disueltos (STD):

$$
\mathrm{ST}=\mathrm{STS}+\mathrm{STD}
$$
(SFT):

Asimismo, los ST incluyen los sólidos volátiles (SV) y los sólidos fijos totales

$$
\mathrm{ST}=\mathrm{SV}+\mathrm{SFT}
$$

De esta manera se puede calcular el porcentaje de sólidos de la fracción orgánica desde sus distintas fracciones: sólidos totales (ST), sólidos volátiles (SV), sólidos fijos totales (SFT), sólidos totales en suspensión (STS), sólidos volátiles en suspensión (SVS), sólidos fijos volátiles (SFV), sólidos totales disueltos (STD), sólidos volátiles disueltos (SVD), sólidos fijos disueltos (SFD).

Las determinaciones de estos sólidos requieren cuidados especiales para no cometer errores debido a la pérdida de carbonato amónico y materia orgánica volátil en la desecación. La determinación se realiza mediante una técnica de gravimetría de acuerdo con el método normalizado (APHA,1989).

Para la determinación de la composición y caracterización físico-química de los residuos e inóculos se analizaran los mismos parámetros mencionados anteriormente, y algunos parámetros adicionales: densidad, humedad, porcentaje de sólidos.

La determinación de las muestras de alcalinidad y $\mathrm{pH}$ se determinó en un equipo COMPACT TITRATOR S+, modelo Crison. La concentración del nitrógeno amoniacal se realizó a través de la técnica volumétrica (APHA - 4500-NorgB). Para ello se utilizó un digestor BLOC-DIGEST 6P (4000629) - SELECTA, un aparato de destilación PRONITRO II (4000627) y una bureta. Finalmente, la estimación de la DQO se realizó por dicromatometría, empleando un método calorimétrico o volumétrico, en este caso se optó por el colorímetro de acuerdo con el método normalizado (APHA - 5220C).

En la determinación de la composición y producción del biogás se utilizaran bolsas Tedlar de muestreo de gases modelo "CALI-5-BOND TM". La composición del biogás fue determinada por lectura en un cromatógrafo de gases SHIMADZU GC-14 B, conectado a un PC para la recogida e integración de datos. Se utiliza una columna de acero inoxidable Carbosieve SII de $2 \mathrm{~m}$. de longitud y $1 / 8$ de pulgada de diámetro. Los reactivos utilizados fueron el gas portador Helio con un caudal de $30 \mathrm{~mL} / \mathrm{min}$ y el gas de calibrado (gases de ensayo padrón de composición conocida de metano, dióxido de 


\begin{tabular}{|l|r|}
\hline Recebido em: 09/02/2007 & HOLOS Environment, v.7 n.1, 2007 - P. 95 \\
\hline Liberado para Publicação em: 15/12/2007 & ISSN:1519-8421 (CD-ROM) / ISSN:1519-8634 (ON-LINE) \\
\hline
\end{tabular}

carbono, nitrógeno, oxígeno y nitrógeno suministrado por Carburos Metálicos S.A.). Así, la determinación del volumen de biogás $(\mathrm{mL})$ se realizó por lectura directa sobre un medidor de flujo de gases por tambor húmedo modelo -RITTER TM Serie TG.

Todas las determinaciones analíticas fueron realizadas según se describe en los Métodos Normalizados (APHA, 1989).

\section{RESULTADOS Y DISCUSIÓN}

\subsection{Caracterización de los Residuos}

Los resultados de la caracterización de los residuos una vez lixiviados, según las técnicas descritas en los Standard Methods - (APHA,1989), sugieren que la presenta una densidad semejante a la estimada para los residuos municipales no compactados (con valores entre 300 y $360 \mathrm{~kg} / \mathrm{m}^{3}$ ), además de un contenido en humedad del 52,6\%, y un porcentaje de ST del 47,4\% en peso. Estos resultados son superiores a los valores de humedad de RSU estimados en España en 1996 (ESPAÑA, 2000); la humedad del RSU oscila entre 40 y $60 \%$ en peso, dependiendo del contenido en materia orgánica fermentable, siendo los valores máximos para los residuos procedentes de mercados (70-80\%) y mínimos para los procedentes de áreas comerciales (10-20\%). El parámetro humedad de los RSU ha decrecido con respecto a épocas pasadas, debido a los mayores porcentajes de productos desechables e inorgánicos. De la misma forma el contenido en materia orgánica ha aumentado en los últimos años, debido a mayores porcentajes de RSU reciclado. No obstante, los estudios de caracterización de los residuos se realizan en base al peso seco, así, los análisis fueron realizados posteriormente al pretratamiento de acondicionamiento (secado, triturado y homogenizado). A continuación en la Tabla 1. se detalla las caracterizaciones físico-químicas iniciales de los residuos utilizados como materias primas: FORSU y LODO. 


\begin{tabular}{|l|r|}
\hline Recebido em: 09/02/2007 & HOLOS Environment, v.7 n.1, 2007 - P. 96 \\
\hline Liberado para Publicação em: 15/12/2007 & ISSN:1519-8421 (CD-ROM) / ISSN:1519-8634 (ON-LINE) \\
\hline
\end{tabular}

Tabla 1- Caracterización físico-química inicial del residuo sólido urbano (FORSU) y del LODO utilizado como materia prima, en los ensayos con reactores a escala de laboratorio y planta piloto.

\begin{tabular}{|c|c|c|c|c|}
\hline \multirow[b]{2}{*}{ Parámetros Analíticos } & \multicolumn{4}{|c|}{ Valores Iniciales } \\
\hline & $\begin{array}{c}\text { FORSU } \\
\text { (reactor } 5 L \text { ) }\end{array}$ & $\begin{array}{c}\text { FORSU } \\
\text { (Planta } \\
\text { Piloto) }\end{array}$ & $\begin{array}{c}\angle O D O \\
\text { (reactor } 5 L)\end{array}$ & $\begin{array}{l}\text { LODO } \\
\text { (Planta } \\
\text { Piloto) }\end{array}$ \\
\hline Densidad $\left(\mathrm{kg} / \mathrm{m}^{3}\right)$ & 295,0 & 361,0 & 1000 & 1083 \\
\hline Materia Orgánica (\%) & 53,3 & 52,6 & 62,5 & 54,2 \\
\hline Humedad $(\%)$ & 17,2 & 19,0 & 96,8 & 96,4 \\
\hline Sólidos (\%) & 82,8 & 81,0 & 3,2 & 3,6 \\
\hline$S T(g / k g)$ & 828,0 & 810,0 & 32,0 & 36,0 \\
\hline$S V(g / k g)$ & 441,0 & 426,0 & 20,0 & 19,5 \\
\hline$S F T(g / k g)$ & 387,0 & 384,0 & 12,0 & 15,7 \\
\hline$S T S(g / L)$ & 17,5 & 8,6 & 3,3 & 8,0 \\
\hline$S V S(g / L)$ & 17,0 & 7,2 & 1,3 & 5,2 \\
\hline$S F S(g / L)$ & 0,5 & 1,4 & 2,0 & 2,8 \\
\hline$P H$ & 7,9 & 7,3 & 7,4 & 7,9 \\
\hline Alcalinidad $(g / L)$ & 0,09 & 0,51 & 0,41 & 0,70 \\
\hline Acidez Total (g/L) & 1,97 & 0,72 & 3,39 & 2,94 \\
\hline $\mathrm{N}-\mathrm{NH}_{4}(\mathrm{~g} / \mathrm{L})$ & 0,28 & 1,06 & 0,78 & 1,79 \\
\hline$N$-total $(\mathrm{g} / \mathrm{kg})$ & 26,0 & 34,0 & 33,7 & 37,5 \\
\hline$C O D(g / L)$ & 39,7 & 64,7 & 37,7 & 24,2 \\
\hline$D Q O(g / L)$ & 41,3 & 73,8 & 41,6 & 29,2 \\
\hline P-total (g/kg TS) & 1,07 & 1,17 & 10,70 & 13,2 \\
\hline$P$-total (\%) & 0,11 & 0,12 & 1,07 & 1,3 \\
\hline$P\left(P_{2} O_{5}\right)(g / k g S T)$ & 0,04 & 0,05 & 0,45 & 0,5 \\
\hline $\begin{array}{l}\text { Carbono }(\%) \quad \text { (Materia } \\
\text { orgánica) }\end{array}$ & 30,9 & 30,5 & 36,3 & 31,5 \\
\hline C:N (Materia orgánica) & 11,9 & 8,9 & 10,8 & 9,2 \\
\hline
\end{tabular}

La FORSU presenta una concentración media de carbono organicp disueltoCOD del orden de 52,2 g/L semejante a los obtenidos por Cecchi et al. (1991) para la FORSU procedente de la Planta S. Giorgio di Nagaro (Italia), que están comprendidos entre 42,8 y 45,0 gCOD/L. La concentración de DQO media es de 57,6 $\mathrm{g} / \mathrm{L}$, aunque en este último caso los valores son bastante variables $(41,3-73,8 \mathrm{~g} / \mathrm{L})$. Los valores de DQO encontrados por Gallert et al (2003) con FORSU procedente de la ciudad de Karlsruhe (Alemania) fueron de $85-120 \mathrm{~g} / \mathrm{L}$, en los ensayos de digestión anaerobia a escala de laboratorio.

El lodo digerido posee un contenido medio en humedad del 96,6\%. Los valores medios de sólidos volátiles fueron $19,75 \mathrm{gSV} / \mathrm{kg}$ y los sólidos fijos del orden de 13,85 


\begin{tabular}{|l|r|}
\hline Recebido em: 09/02/2007 & HOLOS Environment, v.7 n.1, 2007 - P. 97 \\
\hline Liberado para Publicação em: 15/12/2007 & ISSN:1519-8421 (CD-ROM) / ISSN:1519-8634 (ON-LINE) \\
\hline
\end{tabular}

$\mathrm{gSF} / \mathrm{kg}$ por lo que la cantidad de materia biodegradable supone el 54,2\%. Los lodos de EDAR suelen presentar niveles de materia orgánica relativamente altos, entre $36 \mathrm{y}$ $75 \%$, con una cierta cantidad de sales minerales, lo que se refleja en su conductividad.

\subsection{Estudio de Puesta en Marcha y Arranque de un Reactor Tipo Tanque Agitado a Escala de Laboratorio}

El reactor de 5,0 L presenta un volumen útil de 3,0 L, así considerando la densidad de FORSU_T - $750 \mathrm{~kg} / \mathrm{m}^{3}$ y, la fuente de inóculo LODO mesofílico, e imponiendo un $20 \%$ de ST y $30 \%$ de inoculación en cada ensayo, se presenta una descripción del sistema estudiado: 487,5 g de FORSU_T + 0,182 L de agua y 0,78 L de LODO. En la Tabla 2. se presentan las evoluciones temporales de los principales parámetros de control y funcionamiento del reactor (5L) con FORSU.

Tabla 2- Evolución de los principales parámetros de operación en el reactor a escala de laboratorio.

\begin{tabular}{|c|c|c|c|c|c|c|c|c|}
\hline \multirow[b]{2}{*}{ Día } & \multicolumn{8}{|c|}{ Reactor FORSU (5L) } \\
\hline & $\begin{array}{l}S T \\
(\mathrm{~g} / \mathrm{kg}) \\
\end{array}$ & $\begin{array}{l}S V \\
(\mathrm{~g} / \mathrm{kg}) \\
\end{array}$ & $\begin{array}{l}\text { Hidróxido } \\
\text { de sodio } \\
(\mathrm{mL})\end{array}$ & $\begin{array}{l}D Q O \\
(g / L) \\
\end{array}$ & $\begin{array}{l}C O D \\
(g / L) \\
\end{array}$ & $\begin{array}{l}\text { Alcalinid } \\
\text { ad } \\
(\mathrm{mg} / \mathrm{L})\end{array}$ & $p H$ & $\begin{array}{l}\text { Amonio } \\
(\mathrm{mg} / \mathrm{L}) \\
\end{array}$ \\
\hline 2 & 216,4 & 144,0 & 10 & 77,5 & 54,5 & 1500,0 & 7,4 & 1418,5 \\
\hline 4 & 200,0 & 137,6 & 10 & 77,5 & 55,7 & 2350,0 & 8,5 & 1484,6 \\
\hline 7 & 188,7 & 135,8 & 5 & 81,2 & 56,5 & 2440,0 & 8,4 & 1428,0 \\
\hline 10 & 185,2 & 120,0 & 5 & 72,3 & 61,3 & 2143,5 & 8,4 & 1491,8 \\
\hline 14 & 180,0 & 118,4 & 0 & 69,3 & 59,0 & 2220,0 & 7,9 & 1643,0 \\
\hline 17 & 181,0 & 112,0 & 0 & 69,7 & 59,2 & 2455,0 & 7,9 & 1848,0 \\
\hline 21 & 180,0 & 104,0 & 0 & 68,5 & 57,1 & 2578,5 & 8,1 & 2464,0 \\
\hline 24 & 169,0 & 97,6 & 0 & 68,6 & 41,2 & 2450,0 & 8,1 & 2800,0 \\
\hline 28 & 163,0 & 96,0 & 0 & 67,2 & 39,7 & 2218,5 & 8,1 & 3035,2 \\
\hline 31 & 158,0 & 94,4 & 0 & 64,5 & 38,4 & 2445,0 & 8,1 & 2950,0 \\
\hline 35 & 152,0 & 76,8 & 0 & 57,8 & 29,7 & 2667,0 & 8,1 & 2805,0 \\
\hline 38 & 144,0 & 75,2 & 0 & 57,0 & 26,7 & 2770,0 & 8,1 & 2770,0 \\
\hline 42 & 132,0 & 70,4 & 0 & 55,5 & 24,0 & 2984,0 & 8,2 & 2689,0 \\
\hline 45 & 128,0 & 67,2 & 0 & 52,5 & 21,0 & 3250,0 & 8,2 & 2856,0 \\
\hline 49 & 122,0 & 64,0 & 0 & 37,5 & 20,1 & 3525,5 & 8,4 & 2805,0 \\
\hline 56 & 105,0 & 63,2 & 0 & 35,0 & 19,8 & 3201,0 & 8,5 & 2770,0 \\
\hline 60 & 95,0 & 64,0 & 0 & 31,6 & 19,5 & 3150,0 & 8,5 & 2660,0 \\
\hline
\end{tabular}

Según se observa en la Tabla 2, el valor final de eliminación de SV en el reactor (5L) alcanza el 56,1\%, tras 60 días de operación. La eficacia de eliminación de la materia orgánica (expresada como DQO y COD) se presenta más acusada a partir del día 30 de ensayo. Resultados semejantes se obtiene en la eliminación de DQO, un 


\begin{tabular}{|l|r|}
\hline Recebido em: 09/02/2007 & HOLOS Environment, v.7 n.1, 2007 - P. 98 \\
\hline Liberado para Publicação em: 15/12/2007 & ISSN:1519-8421 (CD-ROM) / ISSN:1519-8634 (ON-LINE) \\
\hline
\end{tabular}

59,3\%. Haciéndose una evolución conjunta de las diferentes variables (DQO, COD, $\mathrm{SV}$, alcalinidad, $\mathrm{pH}$, etc.), los resultados parecen indicar que en el reactor $(5 \mathrm{~L})$ con FORSU existe una diferenciación temporal espontánea de las diferentes etapas del proceso: etapa de arranque o hidrólisis (aumento de la COD, crecimiento de la alcalinidad y requerimiento de $\mathrm{NaOH}$ para mantener el $\mathrm{pH}$ ), y etapa de estabilización (aumento de los porcentajes de eliminación de materia orgánica, y estabilización de la alcalinidad y del $\mathrm{pH}$ ).

Las evoluciones tanto del biogás como metano producido, así como sus valores acumulados, se muestran en la Figura 3.

Analizando los datos del biogás, se han podido apreciar los siguientes resultados: en los primeros días de ensayo se detecta una alta producción de biogás y metano. La primera etapa del proceso (arranque) ocurre en la primera semana del ensayo y se caracteriza por una alta producción de biogás (media de 3,3 L/día). Posteriormente el reactor permanece estable hasta el día 40 con una producción baja de biogás (media de 3,28 L/día) y metano $(1,0 \mathrm{~L} /$ día). Con respecto a la producción acumulada de biogás y metano, la misma ocurre de forma continuada en toda la experimentación hasta valores de 82,0 L para biogás y 29,9 L para metano. Cuanto a la composición del biogás casi no se detecta producción de hidrógeno, entre los días 1 y 5. El aumento de la producción inicial de metano a partir del día 8-10 coincide con la disminución de dióxido de carbono. Entre los días 5 y 40 de ensayo, se observan valores constantes de metano $(44,8 \%)$ y dióxido de carbono $(55,2 \%)$, característico de la fase metanogénica. En este caso, la diferencia cronológica entre las fases acidogénica, acetogénica y metanogénica no es fácilmente visible y el reactor se muestra típicamente metanogénico en todo el proceso.

\subsection{Estudio de Transferencia Tecnológica Mediante Ensayo Realizado a Escala de Planta Piloto.}

La planta piloto de 300,0 L presenta un volumen útil de 180,0 L, considerando la densidad de FORSU - $361 \mathrm{~kg} / \mathrm{m}^{3}$ y la fuente de inóculo LODO mesofílico, e imponiendo un $20 \%$ de ST y $30 \%$ de inoculación en cada ensayo, se presenta la descripción del sistema estudiado: 36,0 L de FORSU $(13,0 \mathrm{~kg})+101 \mathrm{~L}$ de agua y 43,0 L de LODO. En la Tabla 3. se presentan las evoluciones temporales de los principales parámetros de control y funcionamiento de la planta piloto con FORSU sin pretratamiento. 


\begin{tabular}{|l|r|}
\hline Recebido em: 09/02/2007 & HOLOS Environment, v.7 n.1, 2007 - P. 99 \\
\hline Liberado para Publicação em: 15/12/2007 & ISSN:1519-8421 (CD-ROM) / ISSN:1519-8634 (ON-LINE) \\
\hline
\end{tabular}

Tabla 3. Evolución de los principales parámetros de operación del reactor FORSU $(300,0 \mathrm{~L})$.

\begin{tabular}{|c|c|c|c|c|c|c|c|c|}
\hline \multirow[b]{2}{*}{ Día } & \multicolumn{8}{|c|}{ Reactor FORSU (planta piloto) } \\
\hline & $\begin{array}{l}S T \\
(\mathrm{~g} / \mathrm{kg})\end{array}$ & $\begin{array}{l}S V \\
(\mathrm{~g} / \mathrm{kg})\end{array}$ & $\begin{array}{l}\text { Hidróxido } \\
\text { de sodio } \\
\text { (mL) }\end{array}$ & $\begin{array}{l}D Q O \\
(g / L)\end{array}$ & $\begin{array}{l}C O D \\
(g / L)\end{array}$ & $\begin{array}{l}\text { Alcalinid } \\
\text { ad } \\
(\mathrm{mg} / \mathrm{L})\end{array}$ & $p H$ & $\begin{array}{c}\text { Amonio } \\
(\mathrm{mg} / \mathrm{L})\end{array}$ \\
\hline 2 & 194,0 & 98,0 & 50 & 54,3 & 50,8 & 352,00 & 7,1 & 3136,0 \\
\hline 4 & 156,0 & 90,0 & 50 & 48,8 & 48,7 & 784,00 & 6,8 & 3024,0 \\
\hline 7 & 136,0 & 86,0 & 50 & 47,6 & 42,6 & 933,60 & 6,9 & 2813,4 \\
\hline 10 & 111,2 & 76,0 & 50 & 45,1 & 40,6 & 1116,00 & 7,4 & 2140,0 \\
\hline 14 & 73,9 & 68,0 & 0 & 43,3 & 36,9 & 1425,00 & 7,5 & 1984,0 \\
\hline 17 & 60,0 & 56,0 & 50 & 39,8 & 34,9 & 1349,00 & 7,9 & 1713,6 \\
\hline 21 & 59,0 & 51,0 & 0 & 37,8 & 32,0 & 1472,50 & 7,9 & 1780,8 \\
\hline 24 & 47,2 & 42,0 & 50 & 35,1 & 28,0 & 1680,00 & 8,4 & 1803,2 \\
\hline 28 & 36,0 & 24,0 & 0 & 33,6 & 24,9 & 1720,95 & 8,5 & 1825,6 \\
\hline 31 & 31,0 & 21,0 & 50 & 32,3 & 23,2 & 1837,50 & 8,6 & 1993,6 \\
\hline 35 & 26,0 & 20,0 & 0 & 31,5 & 22,1 & 1984,50 & 8,6 & 2016,0 \\
\hline 38 & 20,0 & 17,0 & 50 & 29,8 & 20,5 & 2026,50 & 8,7 & 2352,0 \\
\hline 42 & 18,0 & 15,0 & 0 & 25,6 & 19,6 & 2089,50 & 8,7 & 2576,0 \\
\hline 45 & 16,0 & 11,0 & 50 & 21,3 & 18,3 & 1837,50 & 8,8 & 2989,3 \\
\hline 49 & 14,0 & 10,0 & 0 & 19,5 & 17,0 & 1774,50 & 8,9 & 3145,0 \\
\hline 56 & 13,0 & 9,3 & 50 & 16,6 & 15,9 & 1690,50 & 8,5 & 2184,0 \\
\hline 60 & 12,5 & 8,9 & 0 & 15,6 & 12,1 & 1312,50 & 8,3 & 1904,0 \\
\hline
\end{tabular}

Según se observa en la Tabla 3., el reactor piloto que trata FORSU bruta es el que presenta una mayor eficacia de eliminación de materia orgánica expresada como SV, COD y DQO. Así, el valor final de eliminación de SV en el reactor FORSU alcanza el $89,5 \%$. Además se observa una rápida biodegradación (72,1\% de DQO y $75,3 \%$ de COD) tras 60 días de experimentación. Estas cifras están cercanas a los valores de biodegradabilidad que presentan las FORSU procedentes de plantas de tratamiento, según la bibliografía (CHYNOWETH et al., 2001).

Cabe mencionar que el $\mathrm{pH}$ del medio ha sido ajustado a valores neutros mediante la adición de hidróxido de sodio $6 \mathrm{~N}$. Este control ha sido necesario en todos los reactores, para evitar la acidificación del medio. La neutralización permite elevar el $\mathrm{pH}$ al valor óptimo para el crecimiento de los microorganismos formadores de metano y, principalmente, para aumentar la capacidad tampón en la fase de arranque, ayudando a mantener la relación ácidos/alcalinidad.

Debido a problemas operativos la determinación del volumen de biogás generado en la planta piloto no pudo contabilizarse hasta el día 22 de operación, y requirió la sustitución del contador de gas inicialmente instalado por otro modelo (modelo PMMA/PC, por lectura directa con un sensor magnético). Las evoluciones tanto del 


\begin{tabular}{|l|r|}
\hline Recebido em: 09/02/2007 & HOLOS Environment, v.7 n.1, 2007 - P. 100 \\
\hline Liberado para Publicação em: 15/12/2007 & ISSN:1519-8421 (CD-ROM) / ISSN:1519-8634 (ON-LINE) \\
\hline
\end{tabular}

biogás como metano producido, así como sus valores acumulados, se muestran en la Figura 4.

Se puede observar en la figura que, en la etapa de arranque se constata un período pequeño de producción de hidrógeno cercano al $20 \%$, entre los días $1-3$, similar a los valores obtenidos en los previos ensayos realizados en reactores de tanque agitado $(1,1$ L). A partir del día 3, el porcentaje de metano en el biogás asciende rápidamente superando el $50 \%$ a partir del séptimo día de experimentación. Así, entre los días 10 y 22 el volumen de metano equivale a $53,0 \%$ del volumen total de biogás generado coincidiendo con la disminución de los valores del dióxido de carbono, que se sitúan en el 47,0\%. Finalmente, a partir del día 30 de ensayo, el componente mayoritario del biogás es el $\mathrm{CO}_{2}$, que representa más del $70 \%$ del mismo en todo momento. No obstante, se detecta un pequeño aumento del porcentaje de metano entre los días $38 \mathrm{y}$ 50 del ensayo (la producción de biogás en el reactor es superior a 15,0 L/día con una concentración media de metano de $21,2 \%$ ) que puede relacionarse con el aumento detectado, en este mismo período, de los ácidos volátiles y la disminución de los parámetros indicativos del contenido en materia orgánica (SV, DQO y COD). Estos resultados, por tanto, vuelven a indicar que en ese período se produce el ataque a fracciones más resistentes a la biodegradación.
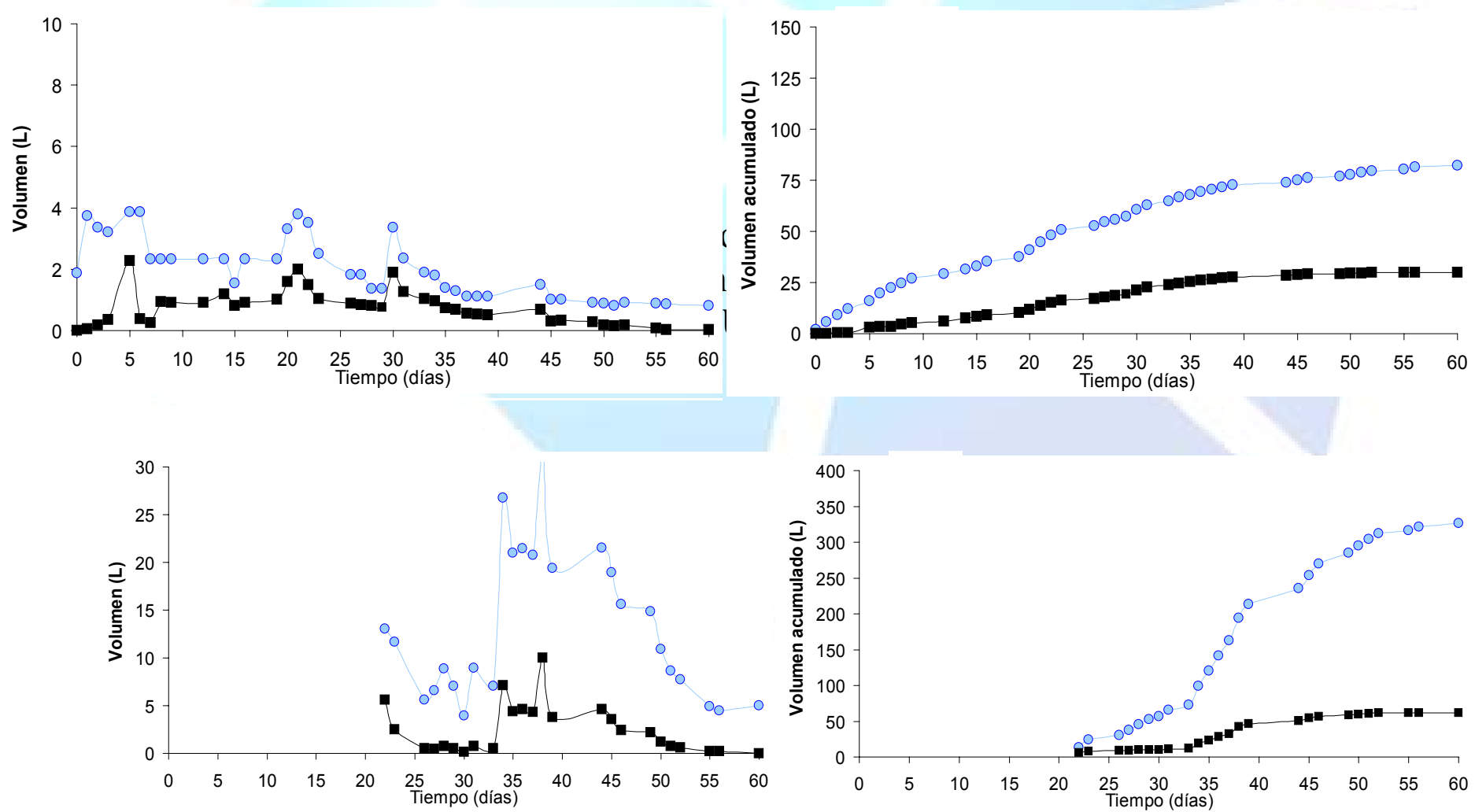

Figura 4. Evoluciones temporales de la producción diaria y del volumen acumulado de biogás y metano en el tratamiento anaerobio seco (20\% de ST y $30 \%$ INÓCULO) del FORSU en planta piloto. 


\section{CONCLUSIONES}

Considerando el objetivo de seleccionar las condiciones operacionales más adecuadas para realizar la etapa de arranque y estabilización de bio-reactores anaerobios, obtener un procedimiento apropiado para el desarrollo de la fase de arranque de la digestión de RSU, en un reactor de tanque agitado a escala de laboratorio y su aplicabilidad a escala de planta piloto", se pueden destacar diversas conclusiones.

Con respecto a la caracterización de residuos sólidos heterogéneos (residuos de restaurante, residuos sólidos urbanos, etc.) se puede concluir que, existe el problema de obtención de una muestra representativa, debiéndose realizar un muestreo sobre lotes homogeneizados y seleccionados al azar para obtener la muestra bruta inicial. Para el manejo posterior del residuo (preparación de las alimentaciones de reactores, etc.) y su caracterización analítica completa se requiere un pretratamiento que mejore su manejabilidad y homogeneidad. El pretratamiento seleccionado consiste en: secar las muestras a $55^{\circ} \mathrm{C}$ durante 24 horas, hasta alcanzar un grado de humedad del $10-20 \%$; a continuación se procede a una homogeneización y reducción del tamaño de partícula hasta 1,0-2,0 cm (reactores tipo SEBAC) o reducción hasta $0,1-0,5 \mathrm{~cm}$ (reactores tanque agitado). Finalmente, se realiza la caracterización de las muestras líquidas o semisólidas sobre el lixiviado obtenido a partir de las muestras sólidas.

El protocolo propuesto para la puesta en marcha de reactores anaerobios operando en condiciones termofílicas $\left(55^{\circ} \mathrm{C}\right)$ y secas $(20 \%)$ y utilizando un porcentaje de inoculación del 30\% con lodos de EDAR digeridos en condiciones mesofílicas ha sido validado en las nuevas condiciones ensayadas, ya que posibilita que los reactores arranquen y alcancen la estabilidad rápidamente, especialmente para la degradación del residuo sin pretratar consistente en la fracción orgánica de residuos sólidos urbanos procedente de la planta de tratamiento de Las Calandrias .

Con respecto a la fracción orgánica del RSU triturada, utilizada en el reactor de 5 Litros, a escala de laboratorio, los resultados indican una puesta en marcha ensayado para este residuo permite alcanzar un arranque efectivo en 50 días, aproximadamente. A lo largo de este período se detecta una primera fase con predomino de la hirólisis y acidogénesis, que alcanza los primeros quince días, y posteriormente se observa un comportamiento menos definido como consecuencia de la coexistencia de todas las etapas del proceso anaerobio. En este sentido cabe destacar que la presencia en el residuo de fracciones de diferente resistencia a la biodegradación se manifiesta en un repunte de la actividad acidogénica en el intervalo comprendido entre los días 30 y 40 de ensayo. Al final del experimento, tras 60 días de operación, los porcentajes de eliminación de la materia orgánica oscilan entre el 55 y el $65 \%$.

Finalmente, los resultados obtenidos en la planta piloto indican que el reactor piloto puede arrancar en un período aproximado de 30 días, mostrando una actividad degradativa adecuada y una rápida estabilización del sistema, para el tratamiento del residuo procedente de la Planta de Tratamiento "Las Calandrias" (FORSU). No obstante, al igual que en el caso anterior, también se observa la existencia de fracciones 


\begin{tabular}{|l|r|}
\hline Recebido em: 09/02/2007 & HOLOS Environment, v.7 n.1, 2007 - P. 102 \\
\hline Liberado para Publicação em: 15/12/2007 & ISSN:1519-8421 (CD-ROM) / ISSN:1519-8634 (ON-LINE) \\
\hline
\end{tabular}

de mayor resistencia a la biodegradación que se degradan en un período aproximado de 50 días. Los porcentajes de eliminación de la materia orgánica alcanzados para este residuo se sitúan, al final del ensayo, en el rango 70-90\%, dependiendo de la forma de cuantificar la materia orgánica (SV, DQO o COD).

Por todo ello, los ensayos con FORSU (triturada y sin triturar) indican que se obtiene un arranque más rápido del proceso y mayores porcentajes de eliminación de la materia orgánica cuando se utiliza el residuo sin triturar, lo que supone una importante ventaja para su aplicación a escala industrial.

\section{AGRADECIMENTOS}

La investigación fue financiada por el Ministerio de Ciencia y Tecnología (MCyT) de España, bajo el proyecto N.PPQ2001-4032 y CTM2004 - 01655.

\section{REFERENCIAS}

APHA - AMERICAN PUBLIC HEALT ASSOCIATION. WATER POLLUTION CONTROL FEDERATION. Standard methods for the examination of water and wastewater. $17^{\text {th }}$ ed. AWWA, WPCF. Washington, DC, 1989.

BIOGAS Works (2005). Internet: www.biogasworks.com

BOLZONELLA, D., INNOCENTI, L., PAVAN, P., TRAVERSO, P., CECCHI, F. Semi-dry thermophilic anaerobic digestion of the organic fraction of municipal solids waste: focusing on the start-up phase. Bioresource Technology, Essex, v. 86, n. 2, p. 123-129, 2003. doi:10.1016/S0960-8524(02)00161-X

BOUALAGUI, H., HAOUARI, O., TOUHAMI, Y., BEN-CHEIKH, R., MAROUANI, L., HAMDI, M. Effect of temperature on the performance of an anaerobic tubular reactor treating fruit and vegetable waste. Process Biochemistry, London, GB, v. 39, p. 2143-2148, 2004.

CECCHI, F., PAVAN, P., MATA ALVAREZ, J., BASSETTI, A., COZZOLINO, C. Anaerobic digestion of municipal solid waste: thermophilic vs. mesophilic performance at high solids. Waste Management \& Research, London, GB, v. 9, p. 305-315, 1991. 


\begin{tabular}{|l|r|}
\hline Recebido em: 09/02/2007 & HOLOS Environment, v.7 n.1, 2007 - P. 103 \\
\hline Liberado para Publicação em: 15/12/2007 & ISSN:1519-8421 (CD-ROM) / ISSN:1519-8634 (ON-LINE) \\
\hline
\end{tabular}

CHYNOWETH, D.P., OWENS, J.M., LEGRAND, R. Renewable methane from anaerobic digestion of biomass. Renew. Energ. 22 (1-2), 1-8.2001.

DE BAERE, L. Anaerobic digestion of solid waste: state of the art. Water Science Technology, Oxford, v. 41, n. 3, p. 283-290, 2000.

DE BAERE, L. (2005). Will anaerobic digestion of solid waste survive in the future? 4Th International Symposium Anaerobic Digestion of Solid Waste. Copenhagen (Denmark), 1:72-82.

EL-FADEL, M., MASSOUD, M. (2001). Methane emissions from wastewater management. Environmental Pollution, 114:177-185.

ESPAÑA. Ministerio del Medio Ambiente. Plan Nacional de Residuos Urbanos (2000-2006). BOE n. 28, de 2 de febrero de 2000.

FORSTER-CARNEIRO, T., FERNÁNDEZ GUELFO, L. A., PÉREZ GARCÍA, M., ROMERO GARCÍA, L. I., ÁLVAREZ GALLEGO, C. J. Optimization of start up phase from municipal solid waste in SEBAC process. Chemical and Biochemical Engineering Quarterly. 18(4): 429-439, 2004a.

FORSTER-CARNEIRO, T., PÉREZ GARCÍA, M., ROMERO GARCÍA, L.I. Digestión anaerobia seca del residuo urbano: funcionamiento del reactor y variables de operación. Tecno Ambiente: Revista Profesional de Tecnología y Equipamiento de Ingeniería Ambiental, Logroño, v.16, n. 161. p. 15-21, 2004b. Disponível em: http://dialnet.unirioja.es/servlet/articulo?codigo=1987161. Acesso em: 20 nov. 2006.

GALLERT, C.; HENNING, A.; WINTER, J. Scale-up of anaerobic of the biowaste fraction from domestic wastes. Water Research, v. 37, n. 6, p. 1433-1441. 2003. doi:10.1016/S0043-1354(02)00537-7.

HARTMANN, H., AHRING, B.K. Strategies for the anaerobic digestion of the organic fraction of municipal solid waste- A review. In: INTERNATIONAL SYMPOSIUM ANAEROBIC DIGESTION OF SOLID WASTE. 4, 2005 , Copenhagen (Denmark),v. 1, p. 34-51.

HERNÁNDEZ, M. (2001). Gestión de lodos en EDARS. META 2001, Ciudad real. 
HOUGHTON, I.T., DING, Y., GRIGS, D. J., NOGUER, M., VAN DER LINDEN, P.J., XIAOSU, D. Climate Change: the scientific basis contribution of working group I. Cambridge University Press, 2001. p. 34-35.

IEA -INTERNATIONAL ENERGY AGENCY (2003). Bio-energy anaerobic digestion activity. biogas from municipal solid waste: an overview of systems and markets for anaerobic digestion of MWS (Booklet). Copenhagen. Minister of Energy Danish Energy Agency.2003.

INE-INSTITUTO NACIONAL DE ESTADÍSTICA. Encuesta sobre recogida y tratamiento de residuos urbanos 2002. Madrid. Librería del INE- Servicios Centrales de Madrid.2002.

ROMERO, L.I., SALES, D., MARTINEZ, E. Comparison of three practical processes for purifying wine distillery wastewater. Process Biochemistry, London, GB, v. 25, n. 3, p. 93-96.1990. 